\title{
Effect of Propranolol and Its Dosages on Maternal-Fetal Heart Rates Coupling in Pregnant Mice and Fetuses
}

\author{
Ahsan H Khandoker ${ }^{1}$, Chihiro Yoshida ${ }^{2}$, Yoshiyuki Kasahara ${ }^{2}$, Kiyoe Funamoto ${ }^{2}$, Yoshitaka Kimura ${ }^{2}$ \\ ${ }^{1}$ Healthcare Engineering Innovation Center (HEIC), Khalifa University, Abu Dhabi, UAE \\ ${ }^{2}$ Tohoku University, Sendai, Japan
}

\begin{abstract}
The aim of this preliminary study is to look how maternal-fetal heart rates and their beat to beat coupling patterns ( $\lambda$ are influenced by injection of $\beta$ blocker(propranolol) into pregnant mice. Total of 9 fetuses from 9 pregnant female mice type of C57BL/6J were divided into three groups [control (3), $\beta$ blockade (3) (4 mg), $\beta$ blockade (3) (10 mg)]. On 17.5-day beat-to-beat maternal and fetal heart rates (MHR and FHR) were simultaneously measured for 20 minutes (10 minutes under normal condition and 10 minutes with saline (to control group) and propranolol (to the $\beta$ blockade groups) solution by using an invasive maternal and fetal electrocardiogram techniques with needle electrodes. Results show that FHR decrease $(p<0.05$ for $10 \mathrm{mg})$ and maternal-fetal heart rate coupling $(\lambda)$ patterns increases for $10 \mathrm{mg} ; p<0.05$ and no significant changes for $4 \mathrm{mg}]$ followed by propranolol infusion (no change with saline). These changes could be caused by propranolol (dosage of $10 \mathrm{mg}$ ) which could have transmitted through the placental barrier. The presented results allow for assessment of the dosages of $\beta$ adrenergic control of maternal and fetal heart, which will further enhance the value of the mouse as a model of heritable human pregnancy and hypertension.
\end{abstract}

\section{Introduction}

Beta-blockers are well known drugs that are frequently used during pregnancy in the treatment of hypertension, cardiomyopathy and maternal-fetal tachycardia $[1,2]$. In complicated pregnancies such as hypertension, treatment with propranolol no congenital abnormalities have been observed, but decreased fetal growth has been reported [3]. Basically, Beta blockers inhibit beta1 receptors in the heart inhibiting activity of the sympathetic nervous system resulting in reduced heart rate and contractility. The main benefit of beta blockade is that it reduces myocardial oxygen demand and catecholamine release which in turn decreases blood pressure [4].

Mouse models with drug manipulations are frequently used to understand how maternal factors influences fetal development [5]. The advantage of pregnant mouse model is that mouse fetus develops quickly, taking only three weeks to a fully-grown mouse [5]. There are clear needs of mouse models and related technology how to assess fetal cardiovascular system in utero of pregnant mice. In our previous studies [6, 7], we demonstrated the procedure to monitor beat to beat heart rate variability (HRV) associated with developing fetal autonomic nervous system in utero by Electrocardiography (ECG) approach. Then that was followed by further investigation on finding evidence of maternal fetal heart rate coupling under saline and atropine infusion [7].

Till now there have been no studies to look at how the maternal and fetal heart rates are influenced by the dosages of $\beta$ blocker in pregnant mice models and if there is any influence of maternal heart rates on fetal heart rates by using coupling analysis technique. Therefore, the aim of this study is to investigate if maternal-fetal heart rate couplings in mice fetuses are influenced by the dosages of $\beta$ blocker and also how they are regulated by sympathetic nervous system on beat by beat.

\section{Methods}

\subsection{Mice and samples}

The ECG recording system for embryonic mouse was explained in the previous works $[6,7,8]$. The Tohoku University Committee for Safety Management of Animals approved the experimental protocol for this study. Total of 6 female mice of type C57BL/6J with body weight of 16$22 \mathrm{~g}$ were used in this study. Mice were housed in cages under control lighting of 12:12h light-dark cycle and temperature of $\left(22 \pm 2^{\circ} \mathrm{C}\right)$. On the day 17.5 of gestation ECG signals (1000 Hz sampling frequency) of pregnant mice and their fetuses were recorded using biomedical amplifier and recording system (Polymate AP1532®:TEAC, Tokyo, Japan). Saline solutions (100 $\mu \mathrm{l} / 10 \mathrm{~g}$ BW at $37^{\circ} \mathrm{C}$ ) were injected into mother's interscapular subcutis of three cases and sympathetic blockade propranolol (4 and $10 \mathrm{mg} / \mathrm{kg}$ in $100 \mu \mathrm{l} / 10 \mathrm{~g} \mathrm{BW}$ solution at $37^{\circ} \mathrm{C}$ ) into other three cases after 10 minutes of recoding. 


\subsection{Time domain Heart Rate Variability (HRV)}

Maternal and Fetal Heart rate variability (MHRV, FHRV) parameters such as mean heart rate (mean MHR, mean FHR), standard deviation of Normal-to-Normal heart rate data (SD FHR, SD MHR) and the square root of the mean squared difference of the successive heart rate data (rmssd MHR, rmssd FHR) were estimated from 5 minutes before and 5 minutes after the injections of saline or propranolol.

\subsection{Frequency domain Heart Rate Variability (HRV)}

Time-frequency analysis by Short-time Fourier transform (STFT) was applied on MHR and FHR series to check the frequency ranges affected by saline or propranolol injections. Twenty minutes' samples of time series were processed with a kaiser window of 256 samples to segment the data with $50 \%$ overlap. For each applied Kaiser window on 256 samples were used to calculate the spectra. Spectral analysis was then performed on linearly resampled (16 Hz) time series using Burg method [13]. The 256-point fast Fourier transform was repeatedly computed with $50 \%$ overlap between adjacent segments. Then the spectral power of each segment was computed and averaged. Kaiser window was applied to avoid spectral leakage. Subsequently, spectral powers in the frequency range of $0 \sim 1 \mathrm{~Hz}$ and $2 \sim 4 \mathrm{~Hz}$ were obtained by integration [9].

\subsection{Fetal Maternal Coupling Analysis}

The synchronization or coupling between R-peaks of Fetal and maternal ECG was estimated by phase coherence method as described earlier in the previous paper [10]. The instantaneous phase time series was obtained by

$$
\varphi\left(t_{k}\right)=2 \pi\left(t-t_{k}\right) /\left(t_{k+m}-t_{k}\right)+2 \pi k
$$

where $t$ and $t_{k}$ are the times of $\mathrm{R}$ peak of fetal and maternal ECG signal and $m$ was set at 1 in this analysis. Then the relative phase $\psi\left(t_{k}\right)$ [PSI] in the time window of $t_{w}$ with respect to maternal ECG signal is calculated using the equation

$$
\psi\left(t_{k}\right)=\left[\varphi\left(t_{k}\right) \bmod 2 \pi\right] / 2 \pi
$$

The phase coupling index $\lambda$ was defined by

$$
\begin{gathered}
\lambda\left(t_{j}\right)=\| \frac{1}{N} \sum_{j=k-N}^{k} \cos \left(\psi\left(t_{j}\right) \|^{2}+\right. \\
\| \frac{1}{N} \sum_{j=k-N}^{k} \sin \left(\psi\left(t_{j}\right) \|^{2}\right.
\end{gathered}
$$

where $N$ is the number of heartbeats in time window of $t_{k}-t_{w} / 2 \leq t_{j}<t_{k}+t_{w} / 2$. $\lambda$ ranges from 0 to 1 with $\lambda=1$ the highest synchronization [10]. $\lambda$ was estimated for $\mathrm{f} 1: \mathrm{m} 3)[\mathrm{f}=$ fetal(beat):m=maternal(beat)].

\subsection{Statistical Analysis}

Two-tailed Fisher's exact test was applied on our very small sample size because it uses only contingency table to examine the significance of the associations of between two kinds of classifications rather than the differences in mean values. In this study, first classification was treatment types (Saline/Propranolol_4mg; Saline/Propranolol_10mg) and the second was outcome types (increase/decrease). Increase/decrease was determined from the difference between pre and post values of the parameters. We carried out total 9 Fisher's exact tests for $9 \mathrm{HRV}$ and $\lambda$ parameters individually [Table I]. For each test, rows and columns of $2 \times 2$ contingency table were prepared from 2 treatment types and 2 outcome types. P value was set at $5 \%$ to determine the significance of the associations between treatment and outcome types. Because of the small sample size, differences between the mean levels of the parameters were not calculated in this study.

\section{Results and Discussion}

This experimental study explored the effect of betablockers mice HRV and coupling between maternal and fetal heart beats in time and frequency domains. Fig 1 showed an example that propranolol injected into mother mouse decreased the fetal heart rate (FHR) and altered (increased $\lambda$ ) the phase synchronization/coupling between maternal and fetal heartbeats as compared to $4 \mathrm{mg}$ propranolol. When saline solution was injected as shown in the previous work [7], although an occasional strong coupling in the beginning of the recording was more visible but no noticeable changes are seen in FHR. As far as the fetal HRV is concerned, when propranolol was injected as shown in Fig 1, powers in the frequency range of $(0 \sim 1 \mathrm{~Hz})$ are shown to gradually increase following injection and changes in coupling patterns $(\lambda)$ are clearly observed.

It was interesting to note that high frequency oscillations of maternal HRV in the frequency range of $(2 \sim 4 \mathrm{~Hz})$ slows down. However, this needs further validation on a large sample size. Pre-post increases in SD MHR, RMSSD MHR, power spectral density (PSD) (2 4 $\mathrm{Hz})$ of MHR, decreases in Mean FHR and increases in RMSSD FHR, PSD $(0 \sim 1 \mathrm{~Hz})$ of FHR after sympathetic blockade with propranolol were found to be significantly $(\mathrm{p}<0.05)$ associated with treatment types (Saline/Propranolol) [Table I]. Propranolol did not have a substantial effect on MHR, suggesting marked predominance of sympathetic tone at baseline. Mean $\lambda$ values were found to be significantly associated with injection types (saline/propranolol) but occasional high $\lambda$ values were also noticed during pre-injection period (Fig 3 ) which were not associated with saline or propranolol 
infusion. However, that needs further validation on larger sample sizes. Results of Fisher's exact test are shown in Table I.
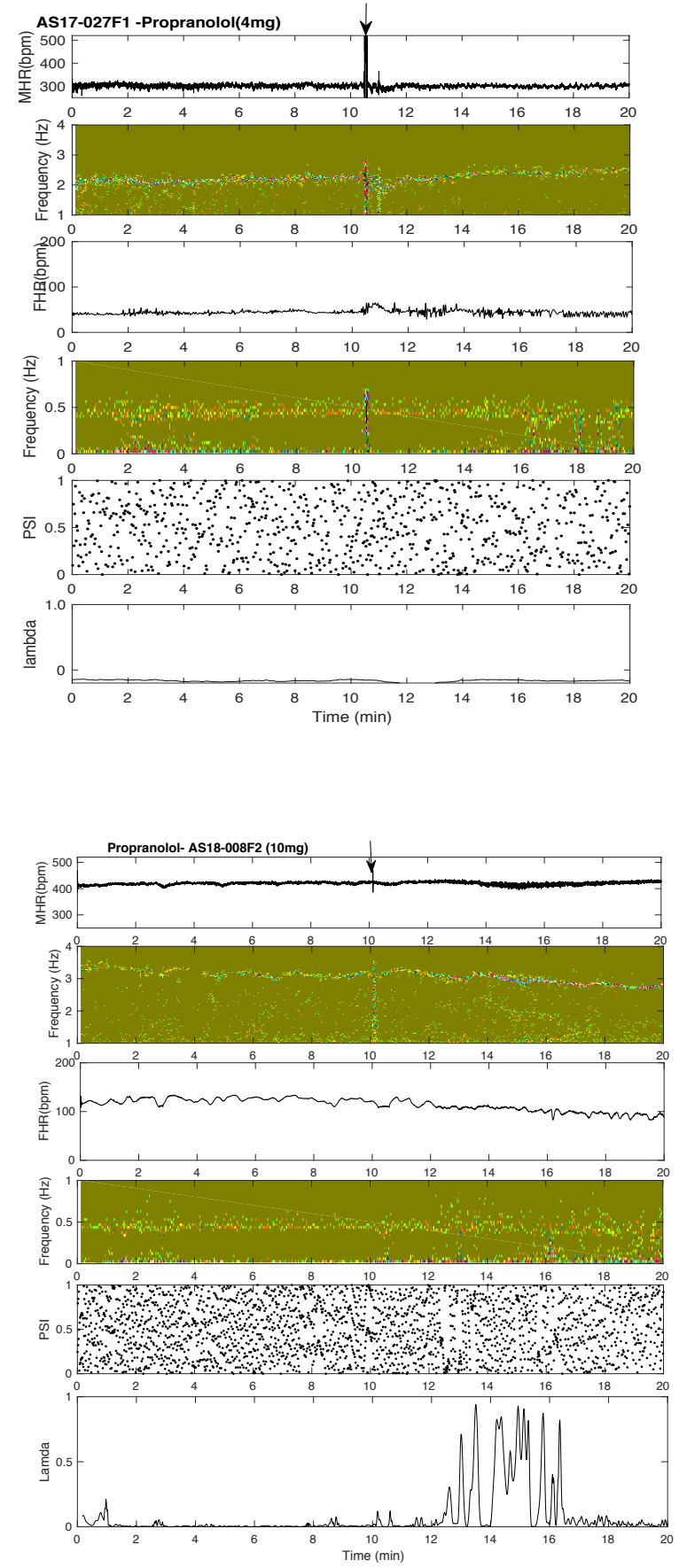

Figure 1. Examples of propranolol treated pregnant mice and its fetuses. psi $\left(\psi\left(t_{k}\right)\right)$, Lamda $\left(\lambda\left(t_{k}\right)\right.$, Fetal heart rate (FHR), Maternal heart rate (MHR). Black arrows indicates the timing of injection. Upper and lower panels show the examples of $4 \mathrm{mg}$ and $10 \mathrm{mg}$ Propranolol administration.

Table 1: Effects of dosages of Propranolol on HRV measures and maternal-fetal heart rate coupling values $(\lambda)$. $\mathrm{PSD}=$ Power spectral density. $*$ significant $(\mathrm{p}<0.05)$ association between outcome types (increase/decrease from pre to post) and treatment types (saline and propranolol).

\begin{tabular}{|c|c|c|c|c|}
\hline & \multicolumn{2}{|c|}{$\begin{array}{l}\text { Propranolol_4mg } \\
(\mathrm{N}=3)\end{array}$} & \multicolumn{2}{|c|}{$\begin{array}{l}\text { Propranolol_10mg } \\
(\mathrm{N}=3)\end{array}$} \\
\hline & Pre & Post & Pre & Post \\
\hline $\begin{array}{l}\text { Mean MHR } \\
(\mathrm{bpm})\end{array}$ & $328 \pm 14$ & $346 \pm 16$ & $421 \pm 15$ & $419 \pm 61$ \\
\hline $\begin{array}{l}\text { SD MHR }(*) \\
(\mathrm{bpm})\end{array}$ & $4.1 \pm 4.4$ & $5.6 \pm 7.2$ & $3 \pm 2.4$ & $6.5 \pm 3.2$ \\
\hline $\begin{array}{l}\text { RMSSD } \\
\text { MHR }\left(^{*}\right) \\
(\mathrm{bpm})\end{array}$ & $3.0 \pm 9.8$ & $3.2 \pm 10$ & $2 \pm 2.4$ & $4.0 \pm 3.5$ \\
\hline $\begin{array}{l}\text { PSD MHR } \\
(*)(2.0-4.0 \\
\mathrm{Hz}) \\
\left(\mathrm{bpm}^{2} / \mathrm{Hz}\right)\end{array}$ & $11.6 \pm 3.2$ & $\begin{array}{l}10.5 \pm 3 . \\
2\end{array}$ & $3.3 \pm 3.2$ & $13.5 \pm 3.2$ \\
\hline $\begin{array}{l}\text { Mean FHR } \\
(\mathrm{bpm})(*)\end{array}$ & $97 \pm 15$ & $101 \pm 17$ & $125 \pm 26$ & $96.0 \pm 40$ \\
\hline $\begin{array}{ll}\text { SD } & \text { FHR } \\
(\mathrm{bpm}) & \end{array}$ & $2.66 \pm 2.1$ & $2.5 \pm 1.2$ & $4.7 \pm 3.8$ & $5.6 \pm 3.0$ \\
\hline $\begin{array}{l}\text { RMSSD } \\
\text { FHR } \\
(\text { bpm) }\end{array} \quad(*)$ & $0.11 \pm 0.3$ & $\begin{array}{l}0.07 \pm 0 . \\
2\end{array}$ & $\begin{array}{l}0.06 \pm 0 . \\
2\end{array}$ & $0.15 \pm 0.8$ \\
\hline $\begin{array}{l}\text { PSD } \quad \text { FHR } \\
(*) \\
(0-1.0 \quad \mathrm{~Hz}) \\
\left(\mathrm{bpm}^{2} / \mathrm{Hz}\right)\end{array}$ & $0.5^{4.74 \pm}$ & $\begin{array}{l}3.23 \\
\pm 0.1\end{array}$ & $\begin{array}{l}11.1 \pm 1 . \\
7\end{array}$ & $48.1 \pm 6.5$ \\
\hline${ }_{(*)}^{\text {Mean } \lambda}$ & $\begin{array}{c}0.06 \pm \\
0.03\end{array}$ & $\begin{array}{r}0.07 \\
\pm 0.01\end{array}$ & $\begin{array}{r}0.05 \\
\pm 0.01\end{array}$ & $0.2^{0.65 \pm}$ \\
\hline
\end{tabular}

The beta receptor antagonist (blockade) caused more effect on fetal heart rate and fetal-maternal heart coupling rather than mean maternal heart rate. The finding that propranolol significantly reduced the power in the frequency range of $0 \sim 1 \mathrm{~Hz}$ in fetal $\mathrm{HRV}$, could be related to sympathetic component of mice fetal HR regulation similar to human HR regulation but 10 times more in the range of low frequency component $0.0 \sim 0.1 \mathrm{~Hz}$ [9].

In human pregnancy, beta blockers were reported to cross the placental barrier and be associated with several adverse effects such as delay intra-uterine growth, respiratory depression, neonatal bradycardia and hypoglycemia, (ie at 12-24 weeks) [11,12]. In mice pregnancy, there are no reports available to date, however, it could also be speculated that beta blockers are transferred across placental barrier as decline FHR was observed. 
The application of standard HRV and phase coupling analysis technique and study protocols to the fetal mice should help discover the role of the autonomic nervous system and beta blocker in HR regulation in fetal mice model and investigate how maternal conditions could affect the coupling between the two independent cardiac rhythms.

Increase in RMSSD MHR (mother) could indicate the effect of beta blocker on maternal HRV by inhibiting sympathetic nervous system activity reflecting on increase in high frequency $(2-4 \mathrm{~Hz})$ powers of PSD MHR. However, it needs further validation to look at how maternal and fetal mice showed antagonistic response to the sympathetic inhibition on lower or higher dosages of beta-blockers. The presented results allow for assessment of the dosages of $\beta$ adrenergic control of maternal and fetal heart, which will further enhance the value of the mouse as a model of heritable human pregnancy and hypertension.

\section{Acknowledgements}

This study was supported by Healthcare Engineering Innovation Center (HEIC) of Khalifa University and a CIRA grant awarded to A.H. Khandoker.

\section{References}

[1] G. Bott-Kanner, A. Schweitzer, SH Reisner, SJ Joel-Cohen, JB Rosenfeld. Propranolol and hydralazine in the management of essential hypertension in pregnancy. Br J Obstet Gynaecol 1980; 87: 110-114.

[2]B. Sandström. Adrenergic beta-receptor blockers in hipertensión of pregnancy. Clin Exp Hypertens B 1982; 1: 127141.

[3] HH. Rotmensch, U. Elkayam, W. Frishman. Antiarrhythmic drug therapy during pregnancy. Ann Intern Med 1983; 98: 487497.

[4] E.P. Widmaier, H. Raff, K.T. Strang, A.J. Vander (2008). Vander's human physiology: the mechanisms of body function.

[5] M .Kaufman. "The Atlas of Mouse Development", in Academic Press, New York, 1992.

[6] A.H. Khandoker, T. Al Khoori, T. Ito, R. Sugibayashi, Y. Kimura. "Assessment of autonomic neurodevelopment in the mouse fetuses by using fetal electrocardiography". In Engineering in Medicine and Biology Society (EMBC), 2016 IEEE 38th Annual International Conference of the 2016 Aug 16 (pp. 2954-2957). IEEE.

[7]A.H. Khandoker, C. Yoshida, Y. Kasahara, K. Funamoto, K. Nakanishi, M. Fukase, Y. Kimura (2018, July). Regulation of maternal-fetal heart rates and coupling in mice fetuses. In 2018 40th Annual International Conference of the IEEE Engineering in Medicine and Biology Society (EMBC) (pp. 5257-5260). IEEE.

[8] Y. Dong, T. Ito, C. Velayo, T. Sato, K. Iida, M. Endo, K. Funamoto, N. Sato, N. Yaegashi, Y. Kimura. "Intrauterine Ischemic Reperfusion Switches the Fetal Transcriptional Pattern from HIF-1 $\alpha$ - to P53-Dependent Regulation in the Murine Brain.
PLoS ONE 9(10): e110577. 2014.

[9] Task Force, "Heart Rate Variability Standards of Measurement, Physiological Interpretation, and Clinical Use". Circulation. 1996; 93: 1043-1065.

[10] K. Niizeki, T. Saitoh. "Cardiolocomotor phase synchronization during rhythmic exercise". The Journal of Physical Fitness and Sports Medicine. 2014 Mar 25;3(1):11-20.

[11] R. Erkkola, R. Lammintausta, P. Liukko, M. Anttila (1982). Transfer of propranolol and sotalol across the human placenta: their effect on maternal and fetal plasma renin activity. Acta obstetricia et gynecologica Scandinavica, 61(1), 31-34.

[12] K. Tanaka, H. Tanaka, C. Kamiya, S. Katsuragi, M. Sawada, M. Tsuritani, T. Ikeda (2016). Beta-blockers and fetal growth restriction in pregnant women with cardiovascular disease. Circulation Journal, 80(10), 2221-2226.

[13] Kay, S. M. Modern Spectral Estimation: Theory and Application. Englewood Cliffs, NJ: Prentice-Hall, 1988.

Address for correspondence.

Dr Ahsan Khandoker

Healthcare Engineering Innovation Center (HEIC)

Department of Biomedical Engineering,

Khalifa University, Abu Dhabi, PO Box 127788, UAE.

E-mail address (optional). ahsan.khandoker@ku.ac.ae 